\title{
Positive Mutation of EGFR Inhibitor Undergo Erlotinib, Gefitinib, Icotinib or Osimertinib Plus Chinese Medicine Versus EGFRTKIs Alone in Patients with NSCLC, a Regional Study East China
}

\author{
Kayembe Mwimbi David ${ }^{1,6}$, Gaochenxi Zhang ${ }^{1,6}$, Zaiwei Huang ${ }^{1}$, Ying Wang ${ }^{1}$, Wenpei Zhu ${ }^{1}$, Dan Wang ${ }^{2}$, \\ Mwema Ngoy Gracia ${ }^{3}$, Wang Hong ${ }^{4}$, Liang Yi ${ }^{5}$, Shuyi Chen ${ }^{6}$, Chen Huihui ${ }^{6}$, Qijin Shu ${ }^{6, *}$ \\ ${ }^{1}$ The First Clinical Medical College of Medicine, Zhejiang Chinese Medical University, Hangzhou, People's Republic of China \\ ${ }^{2}$ Ophthalmology Department, The Clinical University of Kinshasa (UNIKIN), Kinshasa, Democratic Republic of The Congo \\ ${ }^{3}$ The First Affiliated Hospital, College of Medicine, Zhejiang University, Hangzhou, People's Republic of China \\ ${ }^{4}$ Radiotherapy Department, The First Affiliated Hospital of Zhejiang Chinese Medical University, Hangzhou, People's Republic of China \\ ${ }^{5}$ The Third Affiliated Hospital, Zhejiang Chinese Medical University, Hangzhou, People's Republic of China \\ ${ }^{6}$ Department of Oncology, The First Affiliated Hospital of Zhejiang Chinese Medical University, Hangzhou, People's Republic of China
}

Email address:

shuqjiz@163.com (Qijin Shu)

${ }^{*}$ Corresponding author

\section{To cite this article:}

Kayembe Mwimbi David, Gaochenxi Zhang, Zaiwei Huang, Ying Wang, Wenpei Zhu, Dan Wang, Mwema Ngoy Gracia, Wang Hong, Liang Yi, Shuyi Chen, Chen Huihui, Qijin Shu. Positive Mutation of EGFR Inhibitor Undergo Erlotinib, Gefitinib, Icotinib or Osimertinib Plus Chinese Medicine Versus EGFRTKIs Alone in Patients with NSCLC, a Regional Study East China. International Journal of Clinical Oncology and Cancer Research. Vol. 6, No. 2, 2021, pp. 74-89. doi: 10.11648/j.ijcocr.20210602.14

Received: March 19, 2021; Accepted: March 30, 2021; Published: May 15, 2021

\begin{abstract}
Management of non-small cell lung cancer has been changed dramatically since detection of EGFR mutation. The rate of EGFR mutation in East China is unknown. In China, lung cancer is the most frequently diagnosed cancer and the leading cause of cancer-related mortality. The five-year survival rate in patients with lung cancer varies from 3.7-32.9\%, depending on stage and regional differences. The majority of the tumors were Stage IIIB or IV Advanced Lung Adenocarcinoma. The two year survival rate of the patients was $70.6 \%$, the overall survival was $58.8 \%$. Methods: We electronically searched CNKI, Pub Med, Cochrane from database inception and manually searched Chinese-language oncology journals to identify A Positive Mutation of EGFR Inhibitor Undergo Erlotinib, Gefitinib, Icotinib Or Osimertinib Plus Chinese Medicine Versus Erlotinib, Gefitinib, Icotinib Or Osimertinib Alone In Patients With NSCLC, A Regional Study East China. The quality of the included trials was assessed using the method of Cochrane. If heterogeneity existed among subgroups, then overall results (OS) were calculated based on a random-effects model; otherwise, a fixed effects model used. Results: Electronic database searches yielded 1450 citations with NSCLC. We identified full text articles retrieved for detailled evaluation 70. Sample size of each trial had calculated by Rev Man 5.3.
\end{abstract}

Keywords: NSCLC, EGFR Positive Mutation, Erlotinib, Gefitinib, Icotinib or Osimertinib, East China

\section{Introduction}

Lung cancer is the first leading cause of cancer death worldwide in Men. Lung cancer is not only one of the most common malignancies, it also has the highest morbidity and mortality of any cancer. More than $85 \%$ of all lung cancer cases are non-small cell lung cancer (NSCLC) [1]. For a long time, lung cancer was classified as an incurable disease until innovation of new diagnostic and therapeutic tools. Incorporation of diagnostic molecular tools paved a great progress in cancer management particularly in non-small cell lung cancer; NSCLC. Data from the latest global cancer statistics show that there will be 18.1 million new cancer cases and 9.6 million cancer deaths in 2018 [1,2]. Therefore, 
Lungs Cancer Also called Bronchogenic carcinoma with its main types of Non-Small Cell Lungs Cancer (NSCLC 80$85 \%$ ), see figure $1 \&$ Small Cell Lungs Cancer (SCLC 15$20 \%$ ) is most a serious threat to human health and life. NSCLC is characterized by being a highly heterogeneous disease, harboring many genetic aberrations within the various subtypes [2]. More than half of the NSCLC cases are diagnosed at an advanced stage (stages IIIA and IV) [3], and $63 \%$ of cases are 65 years of age or older. Demographics that are shifting toward an older population suggest that oncologists will be seeing more elderly patients with NSCLC in years to come. NSCLC displays several genetic abnormalities including lung cancer with EGFR mutationpositive for epidermal growth factor receptor (EGFR), v-Kiras2 Kirsten rat sarcoma viral oncogene (KRAS) and anaplastic lymphoma kinase (ALK) mutations. Analysis of genes harboring concurrent mutations allowed better understanding of pathogenesis of this disease and hence identifying novel therapeutic targets that improve outcome of lung cancer [4]. In patients with NSCLC, only some percent of the cases harbor treatable drive mutations, such as Epidermal Growth Factor Receptor (EGFR) mutation, for which tyrosine kinase inhibitor can be used. The family of EGFR includes four tyrosine kinase receptors: HER-1 (EGFR), HER-2/ (ERBB2), HER-3 (ERBB3), and HER-4 (ERBB4). EGFR is a trans-membrane receptor; activation of which initiates intracellular signaling cascade with subsequent expression of genes responsible for cell growth, survival, proliferation, differentiation and angiogenesis. It has been reported that deregulation of EGFR signaling pathway is involved in progression of lung cancer [4]. Discovery of EGFR gene has explained why some patients have an excellent response to oral tyrosine kinase inhibitor (TKIs) of this receptor [5]. NSCLC patients with Asian ethnicity, females, none smokers, and adenocarcinoma histology showed the highest clinical benefit when treated with EGFR-TKIs; namely gefitinib and erlotinib [6] while in China at Zhejiang Province hospital, clinically we use Icotinib as first line Egfr-Tkis mutation positive adenocarcinoma Lung Cancer, if resistance of progression disease, a patient will be examine with T790M test and then the use of Osimertinib with brain metastasis plus Chinese medicine in order to reduce the side effect and maintain the Quality of Life. The most frequent mutations of EGFR gene involve exon 18,19 , and 21 . They are either point mutations (in exon 18 and 21) or deletions in (exon 19) clustered around intracellular tyrosine kinase ATP- domain. Analysis of domains of the mutant EGFR revealed or reduced affinity for ATP in EGFR-TKI existence compared to wild type receptor. This existence correlates with efficiency of gefitinib and erlotinib tyrosine kinase inhibitors. Current guidelines NCCN consider EGFR-TKIs as a first-line treatment for patients with advanced NSCLC who harbor EGFR mutation, replacing the conventional cytotoxic chemotherapy. Therefore, evaluation of EGFR mutation is necessary in clinical practice. Data concerning EGFR mutations among NSCLC patient is generally lacking with no available records about status of these mutations in East China.

Chemotherapy is not applied in this case of Adenocarcinoma Egfr Mutation Positive see the NCCN guide line while it's possible to apply Chemotherapy Plus PD-L 1 or PD-1 as Immunotherapy in Adenocarcinoma Egfr Mutation Negative, Shu Qijin et al [6]. To date, Egfr-Tkis remains the standard treatment of care In Stage IIIB-IV adenocarcinoma patients with good functional status. The development of first-, second-, or third-generation epidermal growth factor receptor (EGFR) Tyrosine kinase inhibitors (TKIs) has revolutionized the Treatment of patients with nonsmall cell lung cancer (NSCLC) harboring mutations in the EGFR. However, limited data are available regarding the activity of available EGFR TKIs against uncommon EGFR mutations. Furthermore, epidermal growth factor receptortyrosine kinase inhibitors (EGFR-TKIs) have shown excellent efficacy in advanced EGFR-mutation positive NSCLC, with almost $70 \%$ of response rate. Several randomized controlled trials (RCTs) have confirmed that first-line EGFR-TKI regimen can improve tumor response rate and progression-free survival (PFS) in patients with advanced EGFR-mutant NSCLC. But it still remains appearing to explore whether EGFR-TKI can be applied to neoadjuvant/adjuvant therapy for resectable stages [6], in order to improve (DFS) and overall survival (OS). Upon EGFR-TKIs appearing on the market, some surgeons began recommending TKIs during perioperative period. However, since applications of TKI perioperatively are associated with novel concepts in translational researches, it still remains a highly controversial issue up to now. Different biological characteristics between early and advanced lung cancer can be detected in previous reports and proved by several phenomena. For example, high levels of serum tumor markers, such as carcinoembryonic antigen (CEA), cytokeratin19 fragment antigen 21-1 (CYFRA21-1) and cancer antigen 125 (CA125), are negative prognostic factors in early-stage NSCLC but positive prognostic factors in advanced NSCLC; early stage tumors are more mobile than locally advanced stage NSCLC, which need to be integrated into precise radiotherapy planning [7].

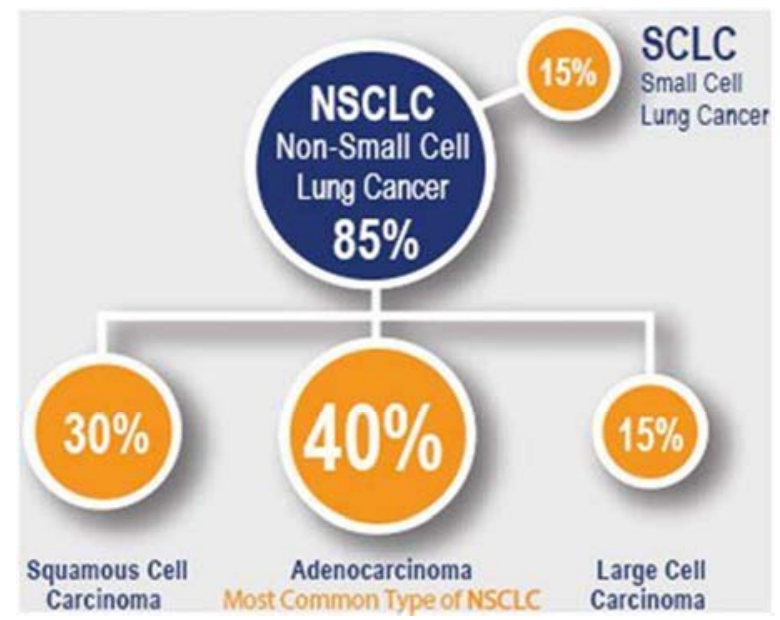

Figure 1. Main types of (NSCLC 80-85\%) \& (SCLC 15-20\%). 


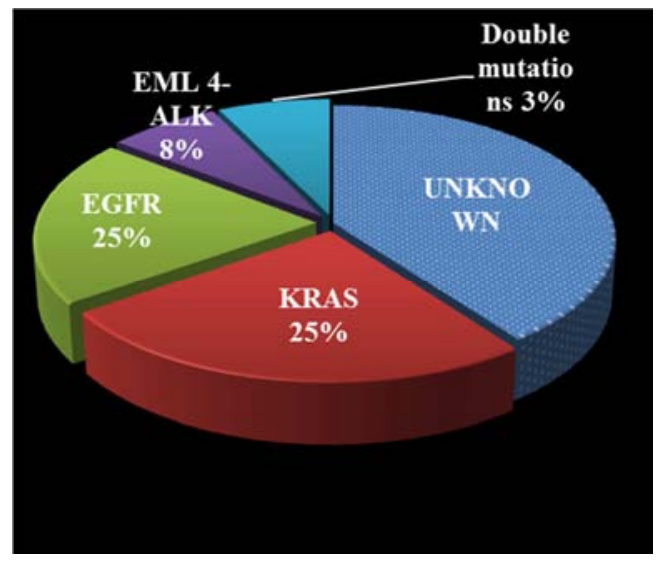

Figure 2. Incidence of mutation-status in NSCLC.

\section{Study Objectives}

This study was conducted to explore the efficacy of A Meta-Analysis of Positive Mutation Of EGFR Inhibitor Undergo Erlotinib, Gefitinib Or Icotinib Plus Chinese Medicine Versus Erlotinib, Gefitinib Or Icotinib Alone In Patients With NSCLC, A Regional Study East China.

Lung cancer is the most common cause of death from cancer worldwide, responsible for nearly one fifth of all cancer deaths (1.59 million deaths, $19.4 \%$ of the total). It is also the most commonly diagnosed and leading cause of death by cancer in men in the United States according for $27 \%$ and $31 \%$ of all cancer death in Women and men, respectively. The median age of diagnosis is 70 years. Although many patients achieve disease-free survival (DFS), some experience a long-term impairment of their quality of life, and disease recurrence is common [7]. Therefore three surveys found that the prevalence of EGFR mutations in NSCLC patients was approximately $10 \%$ in Europe, $17 \%$ in the United States, and $78.8 \%$ in East Asia. The PIONEER study in 2014 was the first to confirm a high frequency of EGFR mutations (51.4\%) in Asian patients with lung adenocarcinoma, with a rate of $62.1 \%$ in China, Taiwan. An epidemiological study in 2015 reported that the EGFR mutation rate among patients with treatment-naïve lung adenocarcinoma in Taiwan was $55.4 \%$, with the main mutations being del $19(44.8 \%)$ and L858R point mutations $(47.9 \%)$.

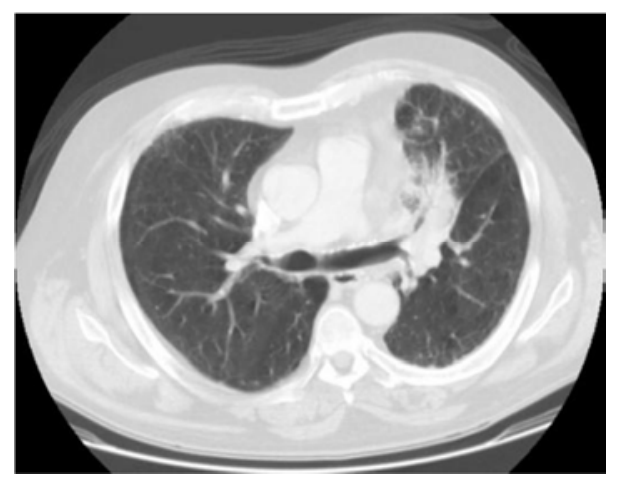

Figure 3. Chest $X$-ray.

\section{Materials and Methods}

\subsection{Tissue Samples}

All the tissue samples were obtained for diagnostic purposes by image-guided tru-cut needle biopsies. Different clinical and imaging parameters were obtained from patients' medical records and all tissue samples were reviewed and verified by a single pathologist. DNA extraction and PCR techniques were conducted at Tissue Culture and Molecular Biology Centre, Zhejiang Province.

\subsection{Egfr Strip Assay}

Mutations of exons number 18, 19, 20 and 21 of EGFR gene were detected by PCR and reverse hybridization technique using EGFR XL StripAssay kit (Vienna Lab Diagnostics). The oligonucleotides probes of EGFR StripAssay target three mutations in exon 18, 24 mutations in exon 19, one mutation in exon 20 and two mutations in exon 21 of EGFR gene. DNA was extracted from formalin-fixed paraffin-embedded (FFPE) tissue sections using DNA FFPE tissue kit (Qiagen) according to manufacturer protocol. The concentration of the extracted DNA was measured by a spectrophotometer before storage at $-20^{\circ} \mathrm{C}$. Specifically hybridized biotinylated oligonucleotides are visualized using streptavidin-alkaline phosphatase and colored substrates.

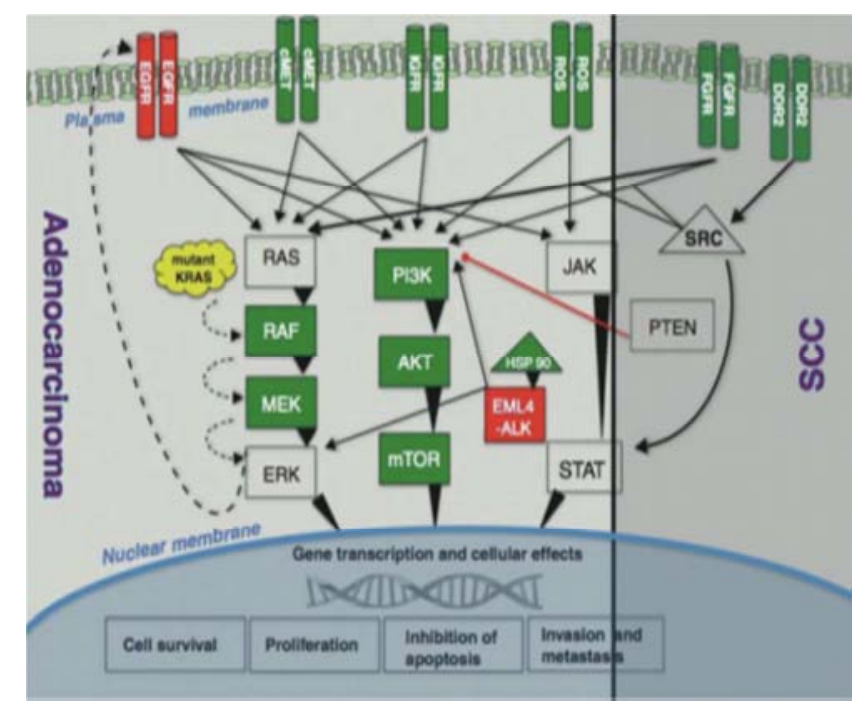

Figure 4. Overview of molecular pathways and potential targets in nonsmall cell lung cancer (NSCLC).

\subsection{Epidermal Growth Factor Receptor (EGFR)}

The epidermal growth factor receptor (EGFR or ErbB1 or HER1) belongs to a family of receptor tyrosine kinases that can trigger a vast array of signalling pathways leading to cell growth, proliferation and survival $(20,21)$. Such flow on pathways include the RAS-RAF-MEK-ERK or MAPK pathway and the PI3K-AKT-mTOR pathways. There are three main mechanisms leading to EGFR activation: increased expression of EGFR on malignant cells; enhanced ligand production by malignant cells; and activating 
mutations of EGFR within malignant cells. EGFR is overexpressed in up to $40-80 \%$ of NSCLC and was a promising translational therapeutic target however it was subsequently discovered that activating mutations rather than overexpression of EGFR was the prime therapeutic target. The two most common mutations are exon 19 deletions $(60 \%)$ and L858R missense substitutions at position $858(35 \%)$ where leucine is replaced by arginine resulting in constitutive activation of the receptor without ligand binding [21-23]. Mutant EGFR can be inhibited either by small molecule TKI (such as gefitinib and erlotinib) or mAb (such as cetuximab). Gefitinib and erlotinib were the first EGFR TKIs to be developed. Both are reversible competitive inhibitors of ATP for the tyrosine kinase domain of EGFR resulting in blockade of downstream pathways.

\subsection{Immunohistochemical Subtyping of the Investigated Tumors}

Histopathological subtype of the investigated tumors was confirmed by evaluation of TTF 1 expression, a tissue marker of pulmonary adenocarcinoma and CK5/6 expression, a tissue marker of squamous cell carcinoma by immunohistochemistry. Four micrometer-thick sections were de-paraffinized in xylene and rehydrated before washing thoroughly in running water. The endogenous peroxidase activity was blocked by incubation of tissue section in dual endogenous enzyme blocking solution (Dako Code K4065, Aligent, USA) for $10 \mathrm{~min}$ followed by washing in running water. Antigens were retrieved by boiling tissue sections in citrate buffer (10mM, pH 6.0) using a microwave for $10 \mathrm{~min}$. The sections were washed in phosphate-buffered saline (PBS) pH 7.6 before incubation with either mouse monoclonal antihuman TTF1 antibody (Dako, clone 8G7G3/1, Aligent, USA) or mouse monoclonal anti-human CK5/6 antibody (Dako, clone D5/16 B4, Aligent, USA) for one hour at room temperature. After washing in PBS, the sections were incubated with Peroxidase labeled polymer conjugated to goat anti-mouse immunoglobulin for $30 \mathrm{~min}$ at room temperature. The sections Cancer Research Journal were washed with PBS and exposed to a freshly prepared 3,30diaminobenzidine tetrahydrochloride (DAB) solution for 510 minutes to visualize brown deposits. The sections were counterstained by hematoxylin, dehydrated in graded alcohol and mounted as usual. TTF1 was visualized as nuclear brown staining while $\mathrm{CK} 5 / 6$ was demonstrated as cytoplasmic brown staining.

\subsection{Study Design and Treatment}

Pathologically, there are 100 types of cancer, including lung cancer, breast cancer, skin cancer or Kaposi sarcomas, colon cancer, Ovarian Cancer, Cervical Cancer, Hepatobiliary Cancer, Pancreas Cancer, prostate cancer, and lymphoma. Cancer starts when a cell is somehow altered so that it multiplies out of control. A tumor is a mass composed of a cluster of such abnormal cells. Most cancers form tumors, but not all tumors are cancerous. Researchers are attempting to find screening tests that may help to dignose lung cancer early, such as Sputum cytology, CT, Imaging tests, and tissue samples (Biopsy) see table 1.

SCLC is more responsive to Chemotherapy than NSCLC. While NSCLC. Furthermore, staging lung cancer is based on whether the cancer is local or has spread from the lungs to the lymph nodes or other organs. Because the lungs are large, tumors can grow in them for a long time before they are found. Even when symptoms - such as coughing and fatiguedo occur, people think they are due to other causes. For this reasons, early-stage lung cancer (stage I \& II) is difficult to detect. Staging in Small Cell Lung Cancer, as limited stage, in this stage, cancer is found on one side of the Chest, involving just one part of the lung and nearby lymph nodes. Extensive stage, in this stage, cancer has spread to other regions of the Chest or other parts of the body. While, stages of Non-Small Cell Lung Cancer as known, stage I, the cancer is located only in the lungs and has not spread to any lymph nodes. Stage II, cancer is in the lung and nearby lymph nodes. Stage III, Cancer is found in the lung and in the lymph nodes in the middle of the Chest, also described as locally advanced disease. Stage III which has two subtypes, if the Cancer has spread only to lymph nodes on the same side of the Chest where the cancer started, it is called stage IIIA. If the Cancer has spread to the lymph nodes on the opposite side of the Chest, or above the collar bone, it is called stage IIIB. While Stage IV, this is the most advanced stage of lung cancer, and therefore is also described as advanced disease. This is when the cancer has spread to both lungs, to fluid in the area around the lungs, or to another part of the body, such as the liver or other organs [8].

\subsection{Western Medicine Treatment}

The management of lung cancer should primarily be based on the histopathological type, secondarily on the stage. NSCLC represents one treatment group and SCLC another, see the National Comprehensive Cancer Network (NCCN) said our Professor Qijin Shu in China. Accordingly, the treatment will be described separately. Less common lung tumors include neuroendocrine tumors including carcinoids and pleural malignancies, such as mesothelioma. Tyrosine kinase inhibitors (TKIs) are medicines that block signals that tell a cell to grow and divide. This can slow or stop cancer cells from growing. In some cases it can cause the cells to die. Why It Is Used. Tyrosine kinase inhibitors (TKIs) are medicines used to treat many different cancers, such as leukemia and non-small cell lung cancer [9].

In the Department of Medical Oncology, The First Affiliated Hospital of Zhejiang Chinese Medical University, Hangzhou, P. R. China, under the supervision Professor Qijin Shu recommande us to respect the use of NCCN guide line inc case of a number of newer-generation TKIs have been developed for NSCLC with driver mutations, such as EGFR and ALK. And we discuss with professor Shu which agents have recently been approved? [9]

So there are already three drugs approved, two first- 
generation TKI, gefitinib and erlotinib, one secondgeneration TKI, afatinib. While lately, we had a new secondgeneration EGFR TKI, dacomitinib, that was approved because the randomized trials show that it increased PFS over the first-generation TKI and the PFS is around 15 months. And then a bit before, we had approved a thirdgeneration osimertinib, and osimertinib again was compared to third-generation TKI, and again it improves PFS and here it's roughly 19 months. Clinically how should clinicians select between these agents when they're deciding on their front-line therapy?

Recently, we have today two options. Either We can go directly to the last generation which is also call the third generation, because it can inhibit the activating EGFR mutation. It's roughly exome 19 and 21 , but also the main mechanism of resistance, T790M. The first and the second generation are not able to inhibit T790M, so if we use them, it means that we have to be able to re-biopsy the patient. So it's either a tissue or a liquid biopsy always done by Professor Shu in our Department of Medical Oncology, but We need these two to look at the resistance mechanism, T790M. And then We will sequence the TKIs, if We find the T790M mutation, We will give osimertinib always $80 \mathrm{mg}$ 1x1/D 65 years old according to the patient status. So We have two different strategies.

While now, in the ALK-mutant population, what are some of the newer agents that have been developed and approved? In Clinical Trials, We had recently seen the data of somme RCTs articles published that compared the second-generation alectinib and its activity was compared to the first-generation crizotinib, and it improved a lot, it increased a lot the PFS, the hazard ratio is 0.5 , so it's major. It's almost 3 years PFS with alectinib compared to roughly 1 year with crizotinib [9, 10]. And impressively, the brain metastases was really well controlled with alectinib. Patients with brain metastases positive is the big issue. In our Department of Oncology, The First Affiliated Hospital of Zhejiang Chinese Medical University, we found that patients with ALK Mutation positive are few and rare.

So how should clinicians in our Hospital select between those two agents? So while, we as always discuss tolerability with the patients because the toxicity profile of both drugs are different. Then we can discuss the costs if there is a major issue because usually the next generation are a bit more expensive. But in probably an ideal world, We would like to see what is the best for the patient based on the resistance mechanism because we know that this disease is moving, is changing. Depending how it's inhibited by TKI and what we would like to achieve as We start with whatever generation, but We monitor the resistance mechanism and We always adapt the next TKI based on that. In that regard, brigatinib is also a second-generation inhibitor and it has been compared with crizotinib, with the same range of benefit of a ratio for PFS is 0.5 . It has a different toxicity profile compared to alectinib and it inhibits different resistance mutations. So between these two second-generation inhibitor, osimertinib that is already on the market, well in China and wold wide, so therefore we will have a lot to discuss with our patient and it's true that biology should help for these patients see Table $1[10]$.

Table 1. Clinical Drugs \& dosage of EGFER TKIs.

\begin{tabular}{lll}
\hline Generations of EGFR-TKIs & Drugs & Daily dosage \\
\hline \multirow{2}{*}{ First generation Egfr-Tkis } & Erlotinib & $0.25 \mathrm{~g}, 150 \mathrm{mg}, 80 \mathrm{mg}, 150 \mathrm{mg}$ capsules \\
& Gefitinib & $250 \mathrm{mg}$ capsules \\
\multirow{2}{*}{ Second generation Egfr-Tkis } & Afatinib & $20 \mathrm{mg}, 30 \mathrm{mg}, 40 \mathrm{mg}, 50 \mathrm{mg} \mathrm{capsules}$ \\
Third generation Egfr-Tkis & Dacomitinib & $0.25 \mathrm{~g}, 20 \mathrm{mg}, 50 \mathrm{mg}, 70 \mathrm{mg}, 100 \mathrm{mg}$ capsules \\
\hline
\end{tabular}

Patients assigned to TKIs received oral EGFR-TKI Erlotinib (Roche, Switzerland) $150 \mathrm{mg}$, Gefitinib (AstraZeneca, UK) $250 \mathrm{mg}$, or icotinib (Icotinib Beta, China) $125 \mathrm{mg}$ per dose, three times per day; the drug was chosen by the patients $1 \times 1 / \mathrm{d}$ ] plus oral CHM. First generation targeted Egfr 19 del exon and 21 L858R exon mutations. The second generation targeted Egfr 18, 19, 21 exon mutation and ERBB2 amplification. Beyond first-line treatment almost all patients who benefit from EGFR-TKIs will eventually develop clinical resistance [11]. About half of the resistance is explained by the acquired EGFR exon 20 T790M mutations, while the third can solve the resistance of first and second generation induced by Egfr 20 T790 M exon mutation [11].

This study has established a new paradigm: all patients with clinical resistance to first-/second-generation EGFRTKIs should be tested for the presence of T790M mutation as Osimertinib should be offered as standard Third line EGFRTKIs treatment for patients who test positive [11]. Gefitinib (Iressa) approved by the U.S. Food and Drug
Administration (FDA) for first-line treatment of advanced EGFR mutation-positive NSCLC with EGFR exon 19 deletions or exon 21 (L858R) substitution mutations as detected by a FDA-approved test (13 July 2015); the European Commission has granted marketing authorisation for gefitinib for the treatment of adults with locally advanced or metastatic NSCLC with activating mutations of EGFR across all lines of therapy (1 July 2009): many countries in Asia including Japan, Korea, Taiwan, China, India and Thailand approved gefitinib since 2003, later the drug was approved as first line. India had several generic versions of gefitinib available. Erlotinib (Tarceva) received regular approval as monotherapy for the treatment of patients with locally advanced or metastatic NSCLC after failure of at least one prior chemotherapy regimen November 18, 2004, regardless EGFR mutational status. After approval as maintenance. October 18, 2016, the FDA modified the indication for erlotinib for treatment of NSCLC by limiting its use to patients whose tumors have specific EGFR 
mutations. The labeling change applies to patients with NSCLC receiving maintenance or second or later line of treatment. These indications will be limited to those patients whose tumors have EGFR exon 19 deletions or exon 21 L858R substitution mutations as detected by a FDAapproved test. The first-line indication previously was limited to patients with EGFR exon 19 deletions or exon 21 substitution mutations. Many countries in Asia including Japan, Korea, Taiwan, China, India and Thailand approved the drug erlotinib since 2004. Afatinib (Giotrif) FDA was initially approved in 2013 for the treatment of patients with metastatic NSCLC whose tumors have EGFR exon 19 deletions or exon 21 (L858R) substitution mutations as detected by a FDA-approved test and in 2016 for metastatic, squamous NSCLC progressing after platinum-based chemotherapy (histology- oriented indication regardless EGFR mutational status). January 12, 2018, the FDA broadened the indication to first-line treatment of patients with metastatic NSCLC whose tumors have non-resistant EGFR mutations as detected by a FDA-approved test. Afatinib is approved in several markets, including the EU, China, Japan, Taiwan and Canada under the brand name Giotrif, also in the US under the brand name Giotrif and in India under the brand name Xovoltib for patients with distinct types of EGFR mutation-positive NSCLC.

In China Clinically, We always combined EGFRTKIs with Immunotherapy, so therefore, Immune checkpoint inhibitor (ICI) is designed to target an inhibitory immune checkpoint molecule, such as programmed death-ligand 1 (PD-L1) and its receptor, programmed death-1 (PD-1), or cytotoxic Tlymphocyte-associated antigen 4 (CTLA-4) [12]. Agents targeting the PD-1/PD-L1 signaling have shown promising response in NSCLC treatment. Two antibodies (nivolumab and pembrolizumab) [12] to PD-1 and two antibodies (atezolizumab and durvalumab) to PD-L1 have been approved by the US Food and Drug Administration (FDA) and/or European Medicines Agency (EMA) for treatment of NSCLC [12]. Unfortunately, only approximately $20 \%$ patients have positively response to ICIs as monotherapy for NSCLC. Therefore, it is of importance to identify patients who may benefit for immune checkpoint blockade therapy. Currently, four immunohistochemistry (IHC) assays (22C3, 28-8, E1L3N, and SP124) have been registered by FDA as companion and complementary diagnostic assays for detecting the expression of PD-L1 in practice. The introduction of these assays has significantly increased the benefit of anti-PD-1/PD-L1 treatments [12], albeit many challenges in anti-PD-1/PD-L1 therapy remain to be overcome. In present review article, the characteristics of anti-PD-1/PD-L1 antibodies, biomarkers, and companion diagnostic assays for patient identification and the significance of the correlation between PD-1/PD-L1 signaling and other driver oncogenes (EGFR, ALK, KRAS, MET, ROS1) in a combinatory therapy including immune checkpoint blockades and targeted agents were also highlighted.

\subsection{Shu Qijin's Experience of Drugs Which Preventing and Treating Severe Adverse Reactions Induced by in Cancer Was Extracted}

Qi-jin shu is a Professor, currently director of the Department of Thoracic Medical Oncology, The First Affiliated Hospital of Zhejiang Chinese Medical University, Hangzhou, P. R. China. He is a doctoral supervisor both Chinese and Foreign students and is engaged in the combination of Chinese and western medicine clinical in the department of Oncology, teaching and scientific research more than many years while $\mathrm{He}$ is good at, minimally invasive diagnosis and treatment of Lung Cancer, liver cancer, Gastric Cancer, CRC,... common combine Chinese and western medicine treatment of the tumor and $\mathrm{He}$ has more much experience in treating the adverse reactions or severe side effects induced by Molecular Targeted Therapy, Immunotherapy (PD-1 or PD-L 1), Chemotherapy of antitumor treatment especially the extremities syndrome called Hand and Foot Syndromes (HFS), Oral Ulcer, Peripheral Neuropathy, Radioactive Pneumonia, such as Bone Marrow Suppression (BMS) as the original opinion which has accumulated rich clinical experience.

At Zhejiang Provincial Chinese Hospital China, We've seen how Professor Shu Qijin combined both Chinese and Western Medicine treating lung cancer including its adverse reaction or severe side effects of II or III degrees induced by Targeted therapy (EGFRTKIs), or Immunotherapy (PD-1, PD-L 1) such as HFS. Clinically, He said that the CNCC guide line recommend Surgery, Immunotherapy (PD-1, PDL1) combined with molecular targeted therapy are currently the main treatment methods of NSCLC, but they are often accompanied by a variety of severe adverse reactions or severe side effects such as hand-foot syndrome (HFS) as main side effects, oral ulcer, peripheral neuropathy, bone marrow suppression, radiation pneumonia and so on. So therefore, Professor Shu Qijin has deep experience in the treatment of such adverse reactions, and the clinical effect is remarkable. In Chinese opinion, Shu thought that the pathogenesis of tumor diseases are evil toxin, blood stasis, Qi deficiency which caused too much fatigue, Yin and Yang are not balanced.

\subsection{Shouzu Ning Decoction (SND) Hand and Foot Syndrome (HFS)}

Shouzu Ning Decoction (SND) is a molecular target drug in the treatment of common adverse reactions, a typical manifestation of Hand and Foot Syndrome (HFS) that is occurrence erythema on the palms and feet, insensitive, pain, cracking, and desquamation. The U.S. national cancer institute, according to the changes in skin, pain, influence the daily activities such as the HFS is divided into different levels: so the level II - III of HFS will be seriously affected the daily action [13]. Oral ulcers are mainly manifested as erythema, pain, and even ulcers and bleeding in the oral mucosa. According to the acute and subacute classification standards of anti-cancer drugs of the World Health 
Organization (WHO), oral ulcers are divided into 5 degrees, and oral ulcers of III-IV degrees can only enter the fluid diet or cannot eat. In clinical diagnosis and treatment, Professor Shu classified HFS and oral ulcer caused by molecular targeted drugs into the category of "sores" in Traditional Chinese medicine, both of which were caused by heat toxin and blood stasis. Drug toxicity repeatedly damaged the spleen and stomach, affecting its transport, chemical and absorption function. The loss of the extremities of hands and feet in water and grain essence and nourishing showed sensory dullness.

\subsection{Hand-Foot Syndrome (HFS)’s Treatment}

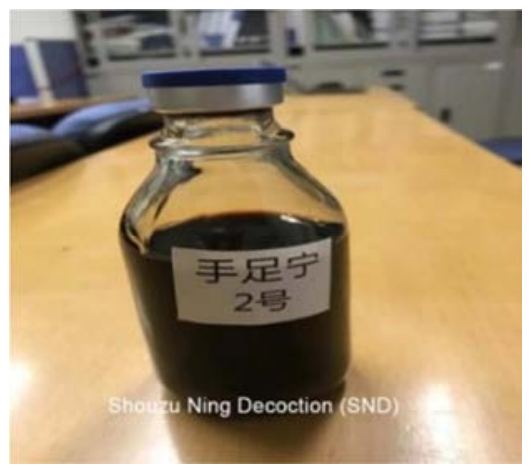

Figure 5. Shouzu Ning Decoction (SND).

Clinically, Shouzu Ning Decoction (SND) (Zhejiang Shouzu Ning Decoction (SND) Group Co. Ltd, Hangzhou,
China) is a botanically sourced, molecularly targeted agent that is prepared as a microemulsion for HFS used.

In China, Chinese Medicine is covered by the National Health Insurance Research Database (NHIRD) and is a generally established form of medical treatment. Chinese Medicine treatment under the NHI is prescribed by boardcertified Chinese medical physicians according to Chinese Medicine syndrome differentiations. The NHIRD is the only computerized reimbursement databases in the world that stores longitudinal prescription data for both Chinese Medicine and Western medicine. This makes the NHIRD an optimal platform to determine the efficacy of Chinese Medicine in reducing the risks of death and disease progression for patients with advanced lung adenocarcinoma treated with first-line or third line EGFR-TKIs (table 1). Due to adverse reactions caused by side effects, Chinese Medicine (CM) is reported as a safe alternative therapy with many roles in improving symptoms, such as reducing cancerrelated fatigue, improving gastro intestinal side effects, protecting liver function, and even ameliorating bone marrow suppression. Chinese Medicine may improves overall survival of lung cancer patients. As a result of Chinese Medicine's advantage in reducing side effects and its potential role in prolonging PFS, Overall Survival (OS), improving the quality of life. In China many elderly patients with advanced NSCLC, always use both Chinese medicine and western medicine [12].

Table 2. Effectiveness of Chinese medicine prolonging PFS \& $O V$.

\begin{tabular}{|c|c|c|c|c|}
\hline Prescription Clinical Trials 2019 & formula & Daily Dosage & formula & dailydosaage \\
\hline \multirow{5}{*}{ CHM used in clinic by Prof Shu Qijin et Al } & Kuangqi & $15 . \mathrm{g}$ & Beishashen & $15 . \mathrm{g}$ \\
\hline & Taizishen & $15 . \mathrm{g}$ & Huanglin & $15 . \mathrm{g}$ \\
\hline & Yujin & 12.g & diaochong & $15 . \mathrm{g}$ \\
\hline & Chuanshidou & 12.g & baixianpi & $15 . \mathrm{g}$ \\
\hline & Maidong & $15 . \mathrm{g}$ & baihe & $15 . \mathrm{g}$ \\
\hline
\end{tabular}

\subsection{Syndrome Differentiation}

Chinese Medicine was chosen from three prescriptions based on TCM syndrome differentiation: a qi-benefiting recipe, a yinnourishing recipe, and a qi-yin benefiting recipe. One experienced TCM physician was assigned to differentiation syndromes and collected the baseline information. TCM diagnostic data and the prescribed medication for 1 month were given at the first visit. The bioactive components were extracted from water. Some quality control markers were selected by referring to the Chinese Pharmacopoeia (2015 edition), and the quantitative analysis was performed by high-performance liquid chromatography coupled with ultraviolet detection (HPLC-UV) [13].

The patients took the Chinese medicine applications on the same day as EGFR-TKIs. The CHM granules were dissolved into $150 \mathrm{ml}$ of warm water to drink twice per day after a meal until the end of the EGFR-TKIs treatment. Clinical research pharmacists took part in and supervised the procedures. EGFR-TKI and CHM treatment lasted until disease progression (DP), unacceptable toxicity, or any other study discontinuation criteria were met. According to previous studies on drug-related toxicities (Melosky and Hirsh, 2014; Califano et al., 2015), dose adjustments or delays were implemented. For EGFR-TKIs-treated patients with serious diarrhea, rashes, or any other EGFR-TKIs-related adverse event (AE), the dosage could be stopped for up to 14 days, and appropriate symptomatic treatment could be provided. EGFR-TKIs were stopped once interstitial lung disease occurred. For CHM-treated patients with abnormal liver function, poor appetite, nausea, diarrhea, and other gastrointestinal AEs, CHM could be delayed up to 14 days. TKIs alone, corresponding to a 7-month improvement in PFS based on previous trials using CHM or TKIs alone in the first-line therapy. The PFS of the second-line EGFR-TKIs targeted therapy was 7 months (Ailing Cao 2017), with an inspection level of $\alpha=0.05$ and power $1-\beta=0.80, \mathrm{HR}=0.50$; here, CHM plus EGFR-TKI may increase PFS to 12 months in the second-line therapy of advanced lung adenocarcinoma with EGFR mutation. PFS is illustrated by the Kaplan-Meier survival curve and log-rank test $80 \%$ [14]. For the PFS and tumor response, an adjusted Cox regression model was used 
to estimate the adjusted hazard ratio (HRs) for differences between the treatment arms with the selected prognostic factors, including the EGFR mutation type, age, sex, EGFRTKIs drugs, treatment line, smoking status, and ECOG PS. Fisher's exact test was used to compare tumor response rates and the incidence of TEAEs between the arms. $\mathrm{P}<0.05$ was considered statistically significant. Analyses were performed using Rev Man version 5.3, SAS version 9.2 (SAS Institute, Cary, NC). Zhejiang Clinical Research Center (ZCRC) experts were invited to manage the statistical analysis.
The results were as follows: calycosin-7-O-beta-Dglucoside $0.012 \%$, icariin $0.075 \%$, psorale isopsoralen $0.067 \%$ in formula I, rosmarinic acid $0.036 \%$, and apigenin $0.0069 \%$ in formula IV, which provided the quality control of the formula. The formulas were applied according to the following regimens (Supplementary Table 3).

Formula I (including tonifying qi and warming yang granules) (Supplementary 1).

FormulaII (nourishing yin granules) (Supplementary 2

Table 3. Traditional Chinese medicine (TCM) syndrome differentiation.

\begin{tabular}{lll}
\hline Traditional Chinese medicine (TCM) syndrome differentiation.* & CHM regimens \\
\hline TCM syndrome differentiation & Main symptoms & Tongue diagnosis \\
\hline 1. Qi syndrome deficiency & $\begin{array}{l}\text { Cough, large amount of sputum, loss of appetite, fatigue and } \\
\text { weakness, pale and bulgy tongue. Secondary symptoms: } \\
\text { spontaneous sweat, unshaped stool, thin superficial, and smooth } \\
\text { pulse }\end{array}$ \\
$\begin{array}{ll}\text { Cough, small amount of sputum, dried mouth, red tongue. Secondary } \\
\text { symptoms: night sweats, insomnia, low fever, thready pulse, rapid pulse } \\
\text { Cough, small amount of sputum, dried mouth, red tongue. Secondary } \\
\text { symptoms: night sweats, insomnia, low fever, thready pulse, rapid pulse } \\
\text { Cough, small amount of sputum, dried mouth, red tongue. Secondary } \\
\text { symptoms: night sweats, insomnia, low fever, thready pulse, rapid pulse }\end{array}$ \\
\hline
\end{tabular}

*Based on the Chinese Medicine New Medicine Clinical Practice Guideline (trial implementation) (published by China Medical Science Press in 2002) and Zhejiang Chinese Medicine Routine Practice (written by the Zhejiang Municipal Commission of Health and Family Planning).

\section{Materials and Methods}

\subsection{Study Design}

This multicenter, randomized, double-blind, placebocontrolled study In A Meta-Analysis of Positive Mutation Of EGFR Inhibitor Undergo Erlotinib, Gefitinib Or Icotinib Plus Chinese Medicine Versus Erlotinib, Gefitinib Or Icotinib Alone In Patients With NSCLC, A Regional Study East China.. All of the patients were given informed consent prior to conducting the study. The study procedures and informed consent form were approved by the Institutional Review Board of The First Affiliated Hospital, Department of Medical Oncology, of Zhejiang Chinese Medical University, Hangzhou, P. R. China. Only randomized controlled trials (RCTs) were eligible for this review; blinding, language, published or not, duration of treatment, and the duration of follow-up were not considered. The RCT is often considered the gold standard for a clinical trial while are often used to test the efficacy or effectiveness of various types of medical intervention and may provide information about adverse effects, such as Drug reaction [15].

\subsection{Random Assignment}

The minimization method was implemented via central randomization on the Internet by a clinical research organization (CRO) (Zhejiang Clinical Research Center, Zhejiang, China); the patients were stratified by sex (male vs. female), age ( $<65$ vs. $\geq 65$ ), ECOG PS (0 vs. 1 vs. 2 ), staging (IIIa vs. IIIb vs. IV), smoking status (yes vs. no), first or second line or third, EGFR mutation status (19 Del vs. 21L858R vs. other rare mutations), TKIs (gefitinib vs. erlotinib vs. Icotinib, Afatinib, Dacomitinib, Osimertinib), and by which center the patient was recruited from.
During the study, clinicians and subjects were blinded to the type of treatment being given.

\subsection{Methods}

\subsubsection{Study Eligibility and Identification}

To identify the studies on the association between EGFR polymorphisms and the survival in NSCLC patients treated with TKI therapy, a systematic search of the available literature according to the Preferred Reporting Items for Systematic Reviews and Meta-Analyses (PRISMA) guidelines for meta-analyses and systematic reviews was performed [15]. All randomized trials of CHM plus EGFR-TKIs (gefitinib vs. erlotinib vs. icotinib) vs the same EGFR-TKIs (gefitinib vs. erlotinib vs. icotinib) alone, and EGFR-TKIs monotherapy vs placebo or best supportive care were eligible for inclusion. Trials were identified from previous meta-analyses. We electronically searched the Medline, Chinese National Knowledge Infrastructure (Chinese language, English 20132021), Pub Med, Cochrane Central Register of Controlled Trails from database inception, CNKI, web of science Wang Fang, and manually searched Chinese-language oncology journals to identify A Meta-Analysis of Positive Mutation Of EGFR Inhibitor Undergo Erlotinib, Gefitinib Or Icotinib Plus Chinese Medicine Versus Erlotinib, Gefitinib Or Icotinib Alone In Patients With NSCLC, A Regional Study East China.. The quality of the included trials was assessed using the method recommended by The Cochrane Collaboration (CC). If heterogeneity existed among subgroups, then overall results (OS) were calculated based on a random-effects model; otherwise, a fixed effects model was used. [16]. Therefore, 70 cases of Non-Small Cell Lungs Cancer among Elderly Patients above 60 years of age were selected and randomly divided into 
two groups: treatment group and control group. Each patient will receive the treatment relevant to their group, respectively. Treatment group (expermental) contained 49 cases and control group 21cases. The treatment group was given the Chinese Medicine plus first or second or third generation Egfr Tkis (gefitinib, erlotinib, icotinib) and control group was given the first generation Egfr Tkis (gefitinib, erlotinib, icotinib) drugs alone.

So furthermore, measurements will be included outcomes...While the articles were searching without restriction to language (English, Chinese) or year of publication from 2010 to 2020 . In fact reviews are defined as systematic if they included an explicit and repeatable methods section describing the search strategy and explicit inclusion/exclusion criteria.

Cochrane collaboration

Cochrane Reviews $(>4,000)$ registered

Identify, appraise and synthesize research-based evidence and present it in accessible

Format; regularly updated

Focus on interventions

Outstanding general resource

Data Sources

Web of Science, We also searched the databases of clinical trials such as Current Controlled Trials (http: //www. controlled-trial.com).

Study Selection

(Inclusion and exclusion criteria)

These types of study review will be confined to randomized controlled trials (RCTs) comparing Chinese Chinese plus first, second or third generation Egfr Tkis versus first, second or third generation Egfr Tkis alone. If the trial states the "randomization" phase, it will be deemed a randomized study, and the blinding will not be restricted.

Inclusion criteria

Age from 30 to 75-years-older.

Patients were eligible for the study if they had primary NSCLC Egfr mutation positive that was confirmed cytologically, pathologically, or by computed tomography

With histologically or cytologically confirmed stage IIIB or IV NSCLC with histologic features of adenocarcinoma.

Patients with a Karnofsky Performance Status (KPS) scores70 and an expected survival time. Finally, patients not treated with chemotherapy or radiotherapy.

Harboring activating EGFR mutations (either exon 19 deletion or L858R in exon 21);

Eastern Cooperative Oncology Group (ECOG) performance status score 0 to 2 .

RCTs

No previous chemotherapy or biologic or immunologic therapy,

Measurable lesion according to response Evaluation Criteria in Solid Tumors (RECIST);

Exclusion criteria

Participants meetings one or more of the following criteria will be excluded:
Not RCTs;

they have a medical history of cardiovascular disease, not Rets

Study with no EGFR mutation analysis

Due to duplicated publication

Severe hypersensitivity to gefitinib or any component of CHM;

Active double cancer

Currently pregnant or breastfeeding

Patient had liver, brain, or bone metastasis (although asymptomatic patients were eligible).

Patients who had just undergone surgery or radiotherapy treatment were also excluded.

\subsubsection{Interventions}

The trial groups which Patients in the treatment group will be received Kanglaite (KLT) injecion, Chinese Medicine plus first generation Egfr Tkis (Gefitinib $250 \mathrm{mg}$ capsules, Erlotinib $150 \mathrm{mg}$ capsules, Icotinib $250 \mathrm{mg}$ capsules) and the control groups received first generation Egfr Tkis (Gefitinib $250 \mathrm{mg}$ capsules, Erlotinib $150 \mathrm{mg}$ capsules, Icotinib $250 \mathrm{mg}$ capsules) alone, regardless of the duration of treatment or follow-up. The basic treatment in both trial and control groups was identical except for KiT injection.(Clinical Trials; CENTRAL), MEDLINE being used the strategy of Dickersin and Larson for controlled clinical trials and EMBASE, CNKI, Pub med. (Excepta Data base) until November 2020.

\subsubsection{Outcome Measures}

The primary endpoint was PFS, which was measured with the date of the videography from a random assignment to the date of objective progression or death by the researcher. In addition, the primary Outcome is same as PFS, which is being assessed from the date of randomization to the earliest sign of disease progression, as determined by Computed tomography or magnetic resonance imaging according to the RECIST criteria [17]. The secondary endpoints included a comparison of Over Survival (OS), Overall Response Rate (ORR), Disease Control Rate (DCR), quality of life (QoL), and safety. In fact, the secondary Outcomes are also MST. MST will be assessed from the date of randomization until $50 \%$ of participants are dead from any cause, with the use of methods that are similar to those used for the analysis of PFS. Tumor response will be assessed every six weeks during the first year after randomization, and every two months after the first year, until disease progression. The follow-up time of both groups is two years after recruitment.

In addition, Computed tomography or magnetic resonance imaging was used to assess tumor at baseline and every 8 weeks until disease progression. Patients who received more than $80 \%$ of the expected dose of EGFR-TKI and CHM were considered adherent. We recorded treatment-emergent AEs (TEAEs) per the Medical Dictionary for Regulatory Activities (version 15.0) and graded them using the National Cancer Institute's common terminology criteria for adverse events (version 3.0). 


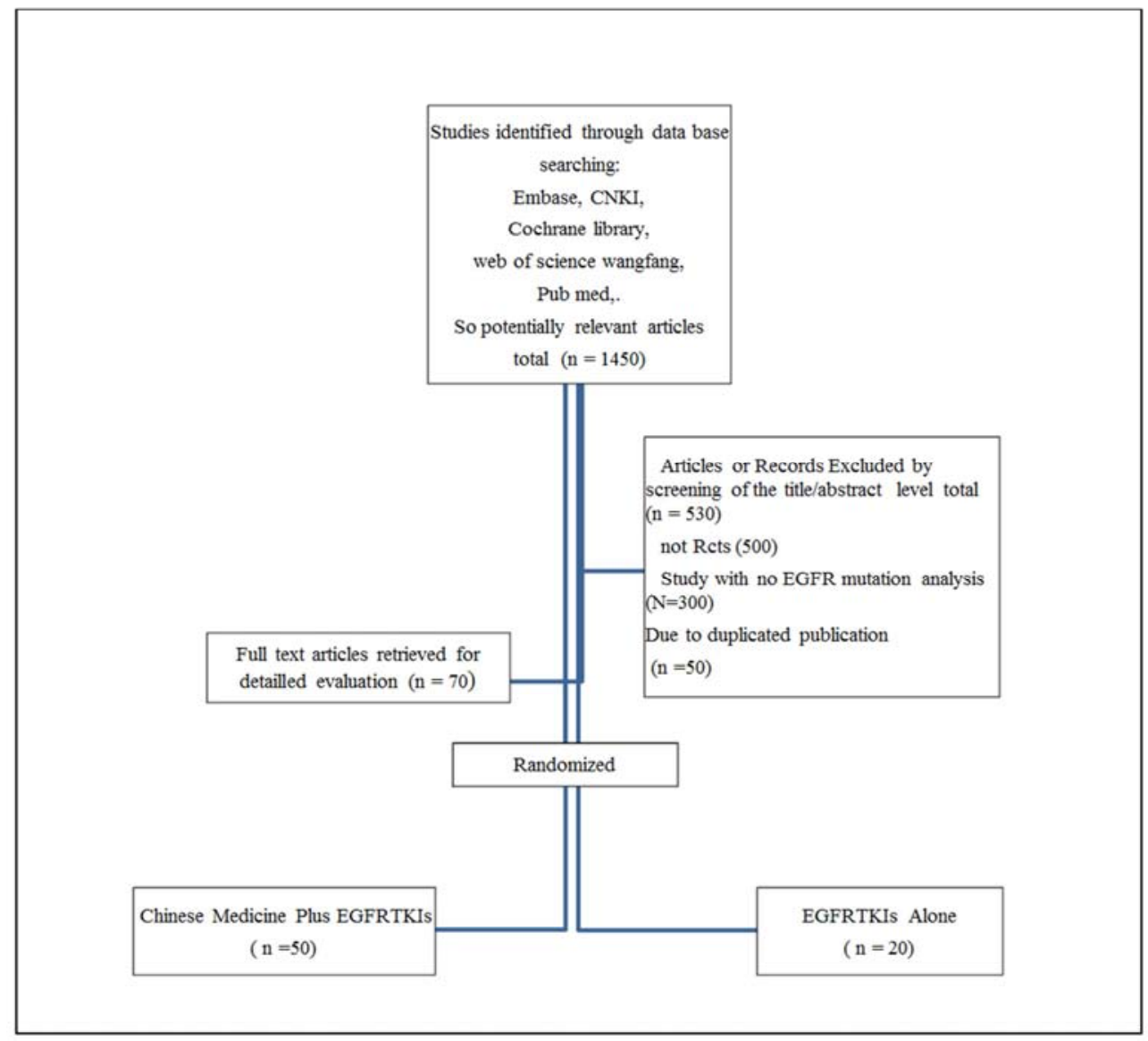

Figure 6. CONSORT diagram: trial profile at the cut-off date for analysis (January 5, 2019) P D, progressive disease.

QoL was collected using the Functional Assessment of Cancer Therapy-Lung (FACT-L) questionnaire (Cella et al., 2002; Thongprasert et al., 2011) and the Lung Cancer Symptom Scale (LCSS) (Hollen et al., 1999).

Quality-of-Life

Improvement Quality of life before and after treatment was assessed using the KPS. Only data for patients whose KPS scores improved by less than 10 points (the minimal clinically significant difference) after treatment were extracted.

\subsubsection{Symptom Improvement}

The percentage of patients exhibiting improvement in the symptoms of cough, hemoptysis, chest pain, fever, fatigue, and anorexia was assessed. We also calculated the percentage of patients whose symptoms resolved completely. Improvement in symptoms was assessed according to the information provided in each included study. Specifically, for the pooled analysis of the trials in which symptom improvement was reported, symptoms were scored according to their degree of severity (ie, grades 1III). For the symptoms of cough, chest pain, and dyspnea, grade I was assigned when the symptoms did not influence daily life, and grade III was assigned when the symptoms were severe, with a marked influence on daily life; symptoms between grade I and III were assigned grade II. For hemoptysis, sputum with blood was assigned grade I, sputum with blood clots or sputum with $\sim 10 \mathrm{mUd}$ of blood was assigned grade II, and extremely bloody sputum or sputum with $>10 \mathrm{mUd}$ of blood was assigned grade III.

\subsubsection{Adverse Events}

Adverse events (AEs) were evaluated at the completion of treatment and included bone marrow suppression (leukopenia, anemia, and thrombocytopenia), nausea and vomiting, phlebitis, hepatic dysfunction, and renal dysfunction. According to the WHO grading criteria for acute and subacute toxicity of anticancer drugs, we only calculated AEs of grades II to IV. If patients withdrew from the study due to an $\mathrm{AE}$, we also included these AEs.

\subsubsection{Quality Assessment}

The quality of the trials was assessed according to the Cochrane Collaboration's criteria11: (1) minimization of selection bias (ie, were the randomization procedure and the allocation concealment adequate); (2) minimization of performance bias (ie, were the patients who received treatment and people who administered the treatment blinded to the interventions); (3) minimization of attrition bias (ie, were withdrawals and dropouts completely described and was the analysis based on intent to treat [ITT\}); and (4) minimization of detection bias (ie, were outcome assessors blinded to the interventions). Based on these criteria, the studies were broadly subdivided into the following 3 categories: $A=$ all quality criteria met, low risk of bias; 
$\mathrm{B}=$ greater than 1 of the quality criteria only partially met, moderate risk of bias; and $\mathrm{C}=$ greater than 1 criteria not met, high risk of bias.

Each trial was assessed independently by one author (Wang) and was checked by another author (Shu). Differences were resolved by discussion.

\subsubsection{Data Extraction}

Data from each included trial were extracted independently by one author (David et al.) and checked by correspondence author (Shu) using a standard extraction form. The form included the following items:

1) General information: published/unpublished; language; authors; article; journal title, year, volume, issue, and page numbers; and funding source;

2) Trial design: predetermined sample size, generation of randomization sequence, allocation concealment method, blinding of information, statistical methods, and attrition;

3) Participants: diagnostic criteria, total number of patients and number of patients in the comparison groups, baseline characteristics (eg, age, gender), inclusion criteria, exclusion criteria, and study settings;

4) Intervention: type of chemotherapy regimen, duration, time, and dose; co-intervention; control; withdrawals, dropouts, and lost to follow-up; and

5) Outcome: outcomes at the end of treatment. The number and type of AEs were also extracted. If the aforementioned data were not available in the trial report, further information was sought by corresponding with the original principal investigator [22].

\section{Statistical Analysis}

Data were analyzed using Meta View 4.2.8 in Review Manager 4.2 (Cochrane collaboration, Oxford, United Kingdom). Meta-analysis was conducted by pooling the different drugs regimens combined together Chinese medicine plus First, second or third Generation Egfr Tkis and comparing these with first, second or third generation Egfr tkis regimens alone for an overall analysis; however, the analysis was divided according to subgroups that were formed based on Chinese medicine plus First, second or third Generation Egfr Tkis regimens. Sensitivity analyses were conducted by excluding low quality trials (David et al.). Analyses were conducted using the ITT principle when possible. Relative risk (RR) was used to analyze dichotomous data. If heterogeneity existed among subgroups, then overall results OR were calculated based on the random effect model; otherwise, the fixed effect model was used [22]. The random effect model was also used to check whether its use might change the direction of the results in cases where heterogeneity was not tested. Heterogeneity was tested using the $\mathrm{Z}$ score and $\mathrm{X} 2$, and $\mathrm{P}<0.1$ was considered statistically significant.

\section{Results}

In summary, Electronic database searches yielded 1540 citations, 70 cases retrieved for detail evaluation of NonSmall Cell Lungs Cancer among Elderly Patients above 70 years of age were selected and randomly divided into two groups: treatment group and control group. Each patient received the treatment relevant to their group, respectively. Treatment groups (expermental) contained 50 cases and control group 20 cases. So therefore, Chinese Medicine plus Egfr Tkis (Erlotinib, Gefitinib, Afatinib, Dacomitinib, or Osimertinib) was effective than plus Egfr Tkis (Erlotinib, Gefitinib, Afatinib, Dacomitinib, or Osimertinib) alone $(\mathrm{P}<0.05)$, the $\mathrm{I} 2=99 \%$ in Mean difference IV Fixed CI $95 \%$ See Figure 7. Comparison on clinical efficacy between the two groups or systematically, a comparison on total effective rate between the two groups showed that data from experimental group (treatment group) was significantly better than those from control group with significant statistical differences $(\mathrm{P}<0.05)$, the $\mathrm{I} 2=99 \%$ in Mean difference IV Fixed CI 95\% see Table 3, [18]

Table 4. Clinical profile of Patients with PFS of EGFR-TKIs treatment $>6$ months.

\begin{tabular}{|c|c|c|c|c|c|c|c|c|c|c|c|c|}
\hline \multirow[t]{3}{*}{ Case } & \multirow[t]{3}{*}{ Sex } & \multirow{2}{*}{\multicolumn{2}{|c|}{$\begin{array}{l}\text { Age } \\
\text { (Years) }\end{array}$}} & \multirow{3}{*}{ Stage } & \multirow[t]{3}{*}{ Smoking } & \multirow[t]{3}{*}{ Gene type } & \multirow[t]{3}{*}{ Sex } & \multirow{2}{*}{\multicolumn{2}{|c|}{$\frac{\text { Age }}{\text { (Years) }}$}} & \multirow[t]{2}{*}{ Stage } & \multirow[t]{2}{*}{ Smoking } & \multirow{2}{*}{ Gene type } \\
\hline & & & & & & & & & & & & \\
\hline & & Mean & SD & & & & & Mean & SD & & & \\
\hline 01 & $\mathrm{M}$ & 66 & 18.1 & IIIB & No & T790M+19delete & $\mathrm{F}$ & 59 & 18.3 & IV & No & S7681 \\
\hline 02 & M & 62 & 17.03 & IV & No & 20 insertion+19del & $\mathrm{F}$ & 56 & 17.4 & IV & No & $\mathrm{T} 790 \mathrm{M}+\mathrm{L} 858 \mathrm{R}$ \\
\hline 03 & M & 64 & 17.5 & IV & Yes & 20insertion & $\mathrm{F}$ & 49 & 15.2 & IV & No & S7681+L858R \\
\hline 04 & M & 47 & 12.9 & IV & Yes & 20insertion + L858R & $\mathrm{F}$ & 63 & 19.6 & IV & No & T790M+G719X \\
\hline 05 & M & 68 & 18.6 & IV & No & T790M+L858R & $\mathrm{F}$ & 55 & 17.1 & IV & No & S76881+19delete \\
\hline Total $\%$ & & 364 & $99.73 \%$ & & & & & 321 & $99.7 \%$ & & & \\
\hline
\end{tabular}

Clinical profile of Patients with PFS of EGFR-TKIs (Erlotinib, Gefitinib or Icotinib) treatment $>6$ months were divided into two groups according to their Gender, Age, Stage \& Gene type. In summary, the sex male were affected 364 in total $99.73 \%$ than the Female 321 in total $99.7 \%$ in different gene types. HR, hazard ratio; ITT, intent to treat;
ORR, overall response rate; OS, over survival; PFS, progression-free survival; $\mathrm{CR}$, complete response; $\mathrm{PR}$, partial response; $\mathrm{SD}$, stable disease; $\mathrm{PD}$, progressive disease; $\mathrm{DCR}$, disease control rate; DoR, duration of response. A two-sided P-value was derived from log-rank test for PFS and from Fisher's exact test for tumor response. 


\section{IPASS: PFS by EGFR Mutation Status}

- Randomized phase III trial; previously untreated pts $(N=1217)$

- PFS: Gefitinib superior to carboplatin/paclitaxel in ITT population

- EGFR mutations strongly predicted PFS (and tumor response) to first-line gefitinib vs carboplatin/paclitaxel

\section{EGFR Mutation-Positive}

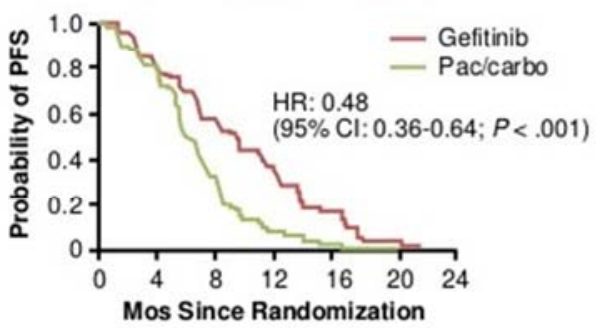

EGFR Mutation-Negative

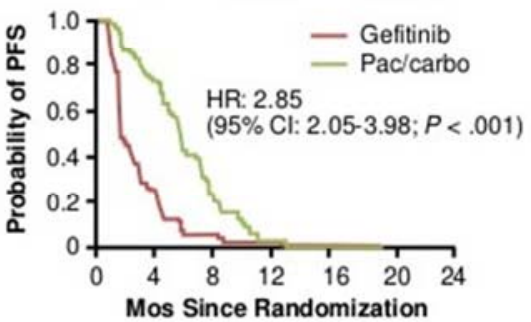

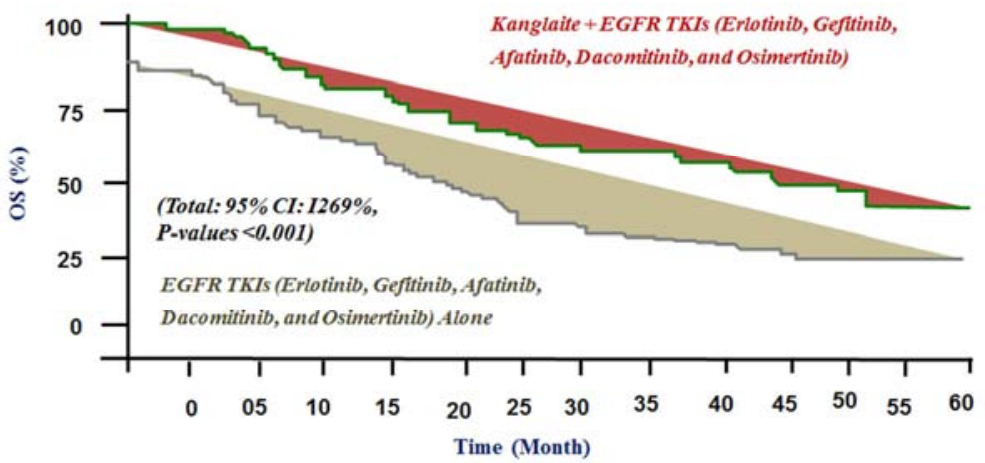

Figure 7. EGFR TKIs (Erlotinib, Gefitinib, Afatinib, Dacomitinib, and Osimertinib) plus Chinese Medicine is significantly benefit than EGFR TKIs Alone.

Table 5. Comparison on Karnofsky and score between two Groups the experimental group and control group.

\begin{tabular}{|c|c|c|c|c|c|c|c|c|}
\hline \multirow{3}{*}{$\begin{array}{l}\text { Authors } \\
\text { Names }\end{array}$} & \multirow{3}{*}{ Year } & \multicolumn{3}{|c|}{ Group I } & \multicolumn{3}{|c|}{ Group II } & \multirow{2}{*}{$\begin{array}{l}\% \\
\text { Percentage \% } \\
\end{array}$} \\
\hline & & \multicolumn{3}{|c|}{$\begin{array}{l}\text { Experimental Group (Chinese Medicine plus } \\
\text { EGFR TKIs Erlotinib, Gefitinib or Icotinib) }\end{array}$} & \multicolumn{3}{|c|}{$\begin{array}{l}\text { Control Group (EGFR TKIs } \\
\text { Erlotinib, Gefitinib or Icotinib) Alone }\end{array}$} & \\
\hline & & Mean & SD & Total & Mean & SD & Total & $\%$ \\
\hline Ailing Cao & 2017 & 65.5 & 5.1 & 70 & 25.6 & 20.1 & 45 & $5.30 \%$ \\
\hline $\begin{array}{l}\text { Haoram } \\
\text { Zhai }\end{array}$ & 2014 & 55.6 & 8.9 & 64 & 24.8 & 19.8 & 79.8 & $4.80 \%$ \\
\hline $\begin{array}{l}\text { Sarah } \\
\text { Burdett }\end{array}$ & 2006 & 28.8 & 3.6 & 32 & 25.5 & 20.4 & 45.9 & $37.50 \%$ \\
\hline Total $\%$ & - & 149.9 & 17.2 & 300 & 75.9 & 60.3 & 170.7 & $47.6 \%$ Total: $95 \%$ CI P-values \\
\hline
\end{tabular}

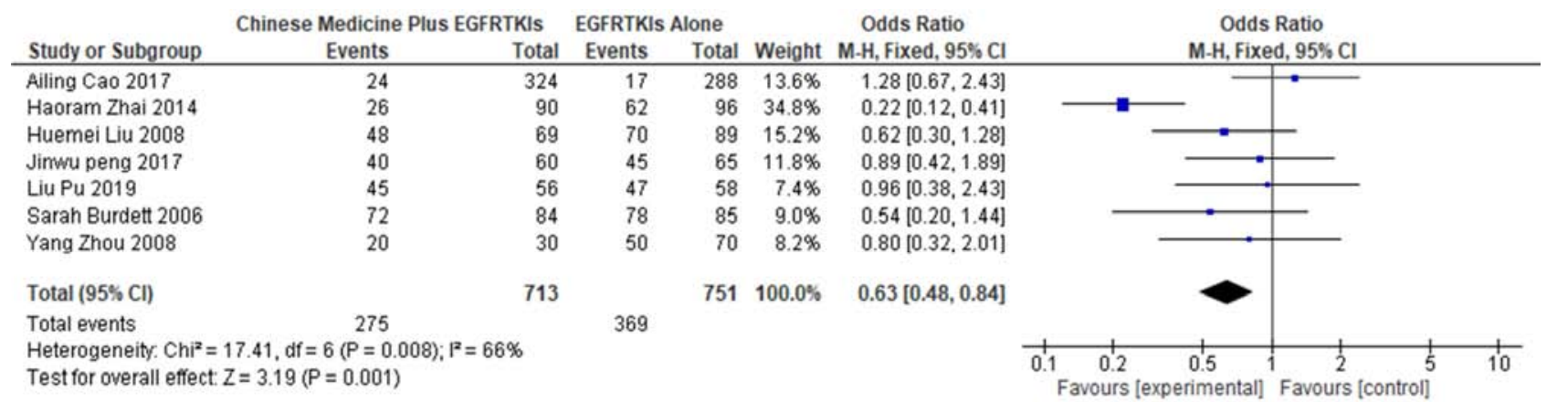

Figure 8. Forest plot of comparison: outcome: Progression Free Survival (PFS). Chinese Medicine (CM) Plus EGFR TKIs (Erlotinib, Gefitinib or Icotinib) Versus EGFR TKIs (Erlotinib, Gefitinib or Icotinib) Alone Risk Ratio (RR, 95\% CI). 


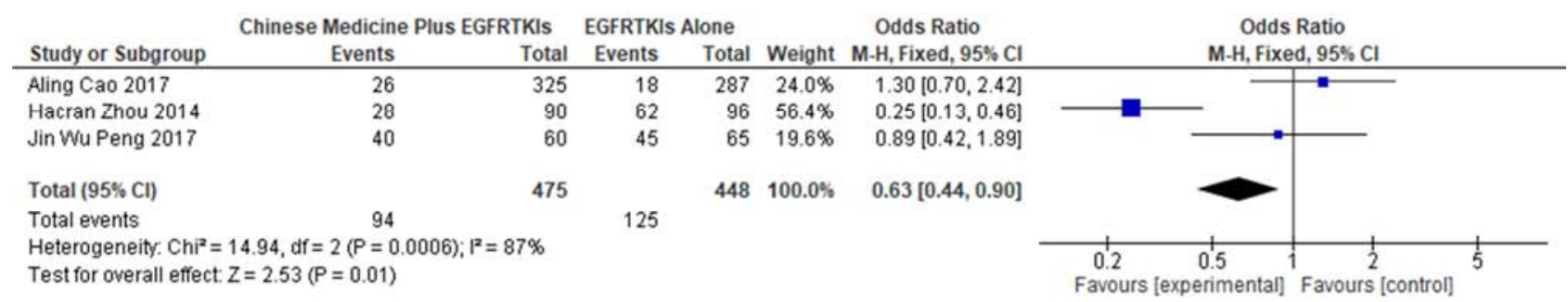

Figure 9. Forest plot of comparison: outcome: Quality of Life (Qol). Chinese Medicine (CM) Plus First-Line Epidermal Growth Factor Receptor (EGFR) Tyrosine Kinase Inhibitors (TKIs) (Erlotinib, Gefitinib or Icotinib) Versus First-Line EGFRTKIs (Erlotinib, Gefitinib or Icotinib) Alone. Std. Mean Different, $95 \% C I)$.

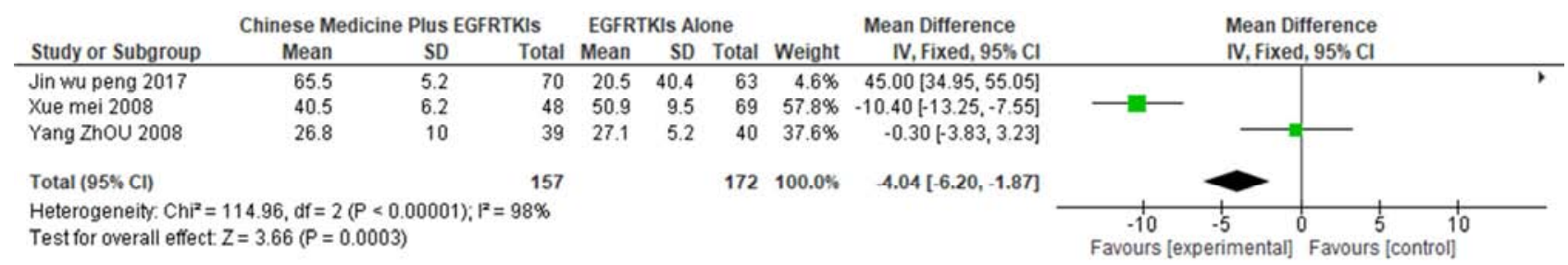

Figure 10. Forest plot of comparison: outcome: Objective Response Rate (ORR). Chinese Medicine Plus EGFR TKIs (Erlotinib, Gefitinib or Icotinib) Versus EGFR TKIs (Erlotinib, Gefitinib or Icotinib) Alone.Std. Mean Difference (MD, Fixed, 95\% CI

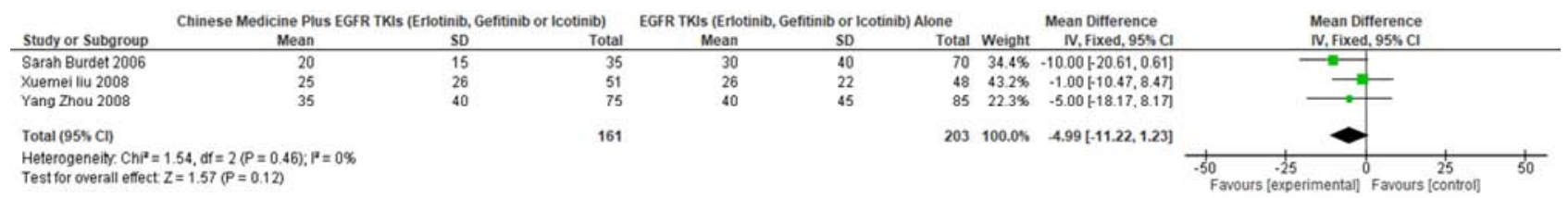

Figure 11. Forest plot of comparison: outcome: Objective Response Rate (ORR). Chinese Medicine Plus EGFR TKIs (Erlotinib, Gefitinib or Icotinib) Versus EGFR TKIs (Erlotinib, Gefitinib or Icotinib) Alone.Std. Risk Ratio (RR, Fixed, 95\% CI).

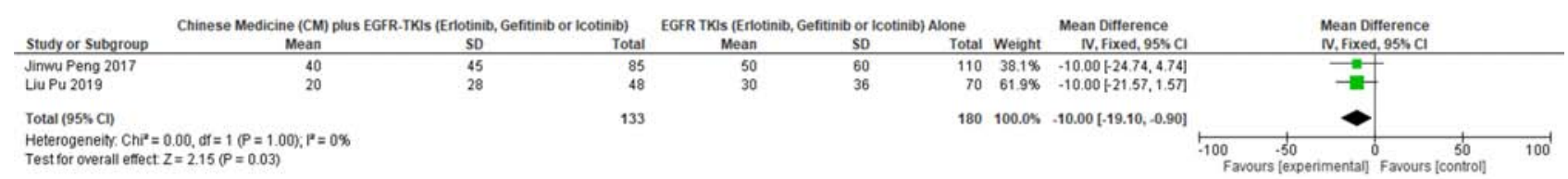

Figure 12. Forest plot of comparison: outcome: disease control rate (DCR). Chinese Medicine (CM) plus EGFR-TKIs (Erlotinib, Gefitinib or Icotinib) Versus EGFR TKIs (Erlotinib, Gefitinib or Icotinib) Alone. Odds Ratio (OR) I2=94\%, P $<0.001$.
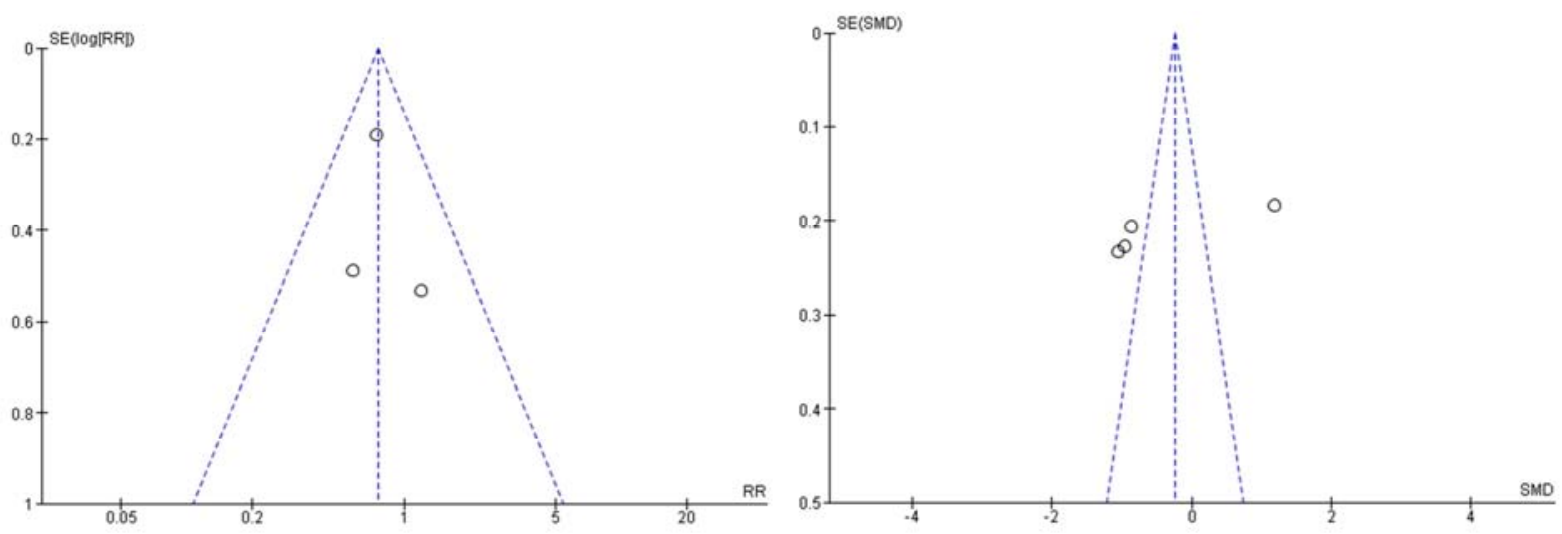

Figure 13. Analysis Funnel plot of comparison: outcome: Progression Free Survival (PFS), disease control rate (DCR), Objective Response Rate (ORR). Chinese Medicine Plus EGFR TKIs (Erlotinib, Gefitinib or Icotinib) Versus EGFR TKI (Erlotinib, Gefitinib or Icotinib) Alone.Std. Mean Difference (SMD).

All HRs and corresponding P-values were unadjusted, except as otherwise noted. HR values for EGFRTKIs (Erlotinib, Gefitinib or Icotinib)+ Chinese Medicine (CM) and EGFRTKIs (Erlotinib, Gefitinib or Icotinib) were derived from a Cox regression analysis, and one-and two-sided P- values were derived from the Wald test from the Cox model. c Adjusted for EGFR mutation type, age, sex, smoking status, ECOG performance status, stage, EGFRTKI drugs (Erlotinib, Gefitinib or Icotinib), Chinese Medicine (CM) syndrome, and prior chemotherapy therapy. $\mathrm{d}$ Defined as the time from 
random assignment to the first date of disease progression. e Defined as the time from random assignment to the date of death from any cause. f Percentages may not add up to $100 \%$ because of rounding. g Defined as the time from the date of the first CR or PR to the first date of progressive disease (per RECIST version 1.1). h Analyzed in the ITT population with CR or PR.

\section{Conclusion}

To the best of our knowledge, to examine the efficacy and safety of A Meta-Analysis of Positive Mutation Of EGFR Inhibitor Undergo Erlotinib, Gefitinib Or Icotinib Plus Chinese Medicine Versus Erlotinib, Gefitinib Or Icotinib Alone In Patients With NSCLC, A Regional Study East China. EGFR TKIs (Erlotinib, Gefitinib Or Icotinib) Plus Chinese Medicine have proved effective and significantly better than EGFR TKIs (Erlotinib, Gefitinib Or Icotinib) alone. EGFR TKIs as first, second or third generation therapy have proved significantly effective for advanced NSCLC but with too much sides effects, reason why we always combined together with Chinese Medicine in order to achieve a better results of treatment and avoid side effects. EGFR mutation is the best established, oncogenic target for management of advanced stage NSCLC. The predictive power of EGFR mutation is confirmed in multiple randomized phase 3 studies comparing first- (Gefitinib or Erlotinib i.e. reversible inhibitors) or second-generation (Afatinib i.e. irreversible inhibitors) or Third generation (Osimertinib).

EGFR TKIs (Erlotinib, Gefitinib Or Icotinib) Plus Chinese Medicine have the improvement in response rate (ORR) and PFS and also are consistent across all age groups, genders, smoking status and performance status (PS). Notably, none of the above studies have shown any benefit in OS for an EGFR-TKIs Plus Chinese Medicine, likely due to the high level of crossover. EGFR-TKIs represent the standard of care as first, second or third-line treatment for advanced EGFR-mutated positive NSCLC. Patients with PS 3-4 may also be offered an EGFR-TKIs as they are likely to receive a similar clinical benefit as patients with good PS. Patients who have benefited from EGFR-TKIs treatment may continue to receive the same therapy beyond initial radiological progression as long as they are clinically stable. The chance to go beyond progression with EGFR-TKI can be considered in patients with oligo- progressive disease, with a consolidation of the progressing lesions with radiation therapy, resulting in a longer PFS. Patients with localized distant progression and ongoing systemic control, continuation of treatment with EGFR-TKI in combination with local treatment of progressing metastatic sites may be considered. A metaanalysis of randomized trials of treatment-native patients reported that EGFR-TKIs statistically significantly prolonged PFS overall, but because of the high rate of crossover at progression, EGFR-TKI had a shorter OS than those who were randomly assigned to chemotherapy (12.8 months, 95\% CI: $11.4-14.3$ vs. 19.8 months, 95\% CI: 17.6
21.7) (Lee et al., 2017). EGFR T790M is a mutation associated with acquired resistance to EGFR-TKI therapy and has been reported in approximately $60 \%$ of patients with disease progression after the initial treatment with erlotinib and gefitinib. Efforts have been made to delay resistance to EGFR-TKI. Osimertinib has shown tobe the potential to prolong PFS to 22.1 months in treatment-naive patients with EGFR advanced NSCLC. However, these strategies overdraft subsequent chemotherapy and targeted therapy and might not benefit OS. Chinese Medicine treatment could be given according to the patients' physical status, syndrome differentiation, and type of cancer treatment. Patients with lung cancer are receiving the theoretical directions of "treatment of cancer by strengthening antipathogenic ability." In China, Chinese Medicine as an adjuvant therapy has shown the potential to reduce EGFR TKIs, chemotherapy toxicity, prolong survival rate, enhance immediate tumor response, and improve Karnofsky performance status (KPS) in advanced NSCLC patients, but its efficacy remains largely unexplored. Chinese Medicine combined with EGFR-TKIs has been demonstrated to be effective in both retrospective and small sample prospective clinical trials. The results of the study conclusively show that Chinese Medicine Plus EGFR TKIs as a first- or second or third-generation therapy provides significantly prolonged PFS and ORR compared with EGFR TKIs alone in Adenocarcinoma with EGFR activating mutations. Condition. The results of this study showed that the total treatment effective rate of the experimental group which using Chinese Medicine plus EGFR TKIs was significantly higher than the control group (EGFR TKIs drugs alone) $(\mathrm{P}<0.05)$, the $\mathrm{I} 2=99 \%$ in Mean difference IV Fixed 95\% CI. The incidence of adverse reactions in patients of the experimental group was lower than that of the control group and the differences between groups were $\mathrm{P}<0.05$, with statistically significant.

In addition, the study with immune indicators (CD3+, $\mathrm{CD} 4+, \mathrm{IgG}$, and IgA) were better than that of the control group, the differences between the groups were $\mathrm{P}<0.05$, have statistically significant, and indicate that the drug can enhance the immune function of cancer EGFR TKIs patients. In the EGFR TKIs of advanced NSCLC, the addition of Chinese Medicine can improve the effect of EGFR TKIs, reduce the side effects of EGFR TKIs, and also significantly improve the immune function of patients.

\section{Funding Source}

This work was supported by the Zhejiang Chinese Medical University.

\section{Declaration of Competing Interest}

The authors declare that there is no conflict of interest associated with the publication of this manuscript. 


\section{Abbreviation}
NSCLC: Non-Small Cell Lung Cancer
EGFR: epidermalgrowthfactorreceptor
TKIs): Tyrosine Kinase Inhibitors
KPS: Karnofsky performance status
PFS: Progression Free Survival
ORR: Overall response rate
OS: Over survival
HR: Hazard ratio
scFv: Single chain variable fragment

SHP2: Srchomology2domaincontaining tyrosine phosphatase SHP-1: Src homology region 2 domain-containing phosphatase-1

TC: Tumor cells

TCR: T cell receptor

TIM-3: Cell immunoglobulin mucin-3

TMB: Tumor mutation burden

TTP: Time to progression.

PD: Progression disease

PD-1 Programmed Death 1

PD-L 1: Programmed Death Ligand 1

HFS: Hand Foot Syndrome

LN: lymph node

SD: Stable disease

PR: Partial response

LAR: long-acting release

NCCN: National Comprehensive Cancer Network

CM: Chemotherapy

TCM: Traditional Chinese Medicine

WM: Western Medicine

\section{References}

[1] American Cancer Society. Lung Cancer (Non-Small Cell). Available at http://www.cancer.org/cancer/lungcancernonsmallcell/detailedguide/non-small-cell-lung-cancersurvivalrates. Updated March 4, 2015. Accessed May 19, 2015.

[2] Ho C, Ramsden K, Zhai Y, et al. Less toxic chemotherapy Improves uptake of all lines of chemotherapy in advanced non-small-cell lung cancer: a 10-year retrospective population-based review. J Thorac Oncol. 2014; 9: 1180-1186.

[3] Li T, Kung HJ, Mack PC, et al. Genotyping and genomic profiling of non-small-cell lung cancer: implications for current and future therapies. J Clin Oncol 2013; 31: 1039-49.

[4] Gao G, Ren S, Li A, et al. Epidermal growth factor receptortyrosine kinase inhibitor therapy is effective as first-line treatment of advanced non-small-cell lung cancer with mutated EGFR: a meta-analysis from six phase III randomized controlled trials. Int J Cancer. 2012; 131: E822E829.

[5] Kim ST, Uhm JE, Lee J, et al (2012). Randomized phase II study of gefitinib versus erlotinib in patients with advanced non small cell lung cancer who failed previous chemotherapy. Lung Cancer, 75, 82-8.

[6] NCCN Clinical Practice Guidelines in Oncology (NCCN Guidelines ${ }^{\circledR}$ ) Non-Small Cell Lung Cancer (Version 3. 2018).
[7] Cella, D., Eton, D. T., Fairclough, D. L., Bonomi, P., Heyes, A. E., Silberman, C., et al. (2002). What is a clinically meaningful change on the functional assessment of cancer therapy-lung (fact-l) questionnaire? Results from eastern cooperative oncology group (ecog) study 5592. J. Clin. Epidemiol. 55 (3), 285-295. doi: 10.1016/S08954356(01)00477-2.

[8] Zhou C, Wu YL, Chen G, et al. Erlotinib versus chemotherapy as first-line treatment for patients with advanced EGFR mutation-positive non-small-cell lung cancer (OPTIMAL, CTONG-0802): a multicentre, openlabel, randomised, phase 3 study. Lancet Oncol. 2011; 12: $735-742$.

[9] Qijin Shu (shuqjhz@163.com) Department of Medical Oncology, Integrated Chinese and Western Medicine, The First Affiliated Hospital of Zhejiang Chinese Medical University, Hangzhou 31000, P. R. China, 12. 2020.

[10] S. Novello, J. Mazières, I. J. Oh et al., "Alectinib versus chemotherapy in crizotinib-pretreated anaplastic lymphoma kinase (ALK)-positive non-small-cell lung cancer: results from the phase III ALUR study," Annals of Oncology, vol. 29, no. 6 , pp. $1409-1416,2018$.

[11] Nguyen KS, Kobayashi S, Costa DB. Acquired resistance to epidermal growth factor receptor tyrosine kinase inhibitors in non-small-cell lung cancers dependent on the epidermal growth factor receptor pathway. Clin Lung Cancer. 2009; 10 (4): 281-9.

[12] S. H. Lim, J. M. Sun, S. H. Lee, J. S. Ahn, K. Park, and M. J. Ahn, "Pembrolizumab for the treatment of non-small cell lung cancer," Expert Opinion on Biological Therapy, vol. 16, no. 3, pp. 397-406, 2016.

[13] Zhang Bei, Hu Pi-li. Effect of integrated traditional Chinese and western medicine on the quality of life of cancer patients [J]. Chinese Journal of Oncology 25 (13): 98-100. Cufer T, Vrdoljak E, Gaafar R, et al (2006).

[14] D. Moher, A. Liberati, J. Tetzlaff, and D. G. Altman, "Preferred reporting items for systematic reviews and metaanalyses: the PRISMA statement," International Journal of Surgery, vol. 8, no. 5, pp. 336-341, 2010.

[15] National Center for Biotechnology Information, U.S. National Library of Medicine, Bethesda, MD, USA, 2019, https://www.ncbi.nlm.nih.gov/pubmed.

[16] P. C. L., Y. F. F., H. Y., et al. Targeting PKC $\delta$ as a therapeutic strategy against heterogeneous mechanisms of EGFR inhibitor resistance in EGFR-mutant lung cancer. Cancer cell 2018; 34: 954-69.

[17] Liu Jiaxiang. Progress in TCM treatment of lung cancer [J]. Chinese Oncology, 2002, 11 (6): 18-21.

[18] Wang Xueqian, Zhang Ying, Liu Jie, Et al. Randomized controlled study of self-made rash granules in treating EGFR-TKI related rash [J]. World Journal of Integrated Traditional Chinese and Western Medicine, 2016, 11 (12): 1629-1632.

[19] Zhejiang Chinese Medical University, S. J. L.. Study on the effect and dynamic changes of syndrome of TCM of Traditional Chinese Medicine combined with Icotinib in treatmeng of advanced NSCLC. 2015. 
[20] Li, S. G., Chen, H. Y., Ou-Yang, C. S., Wang, X. X., Yang, Z. J., Tong, Y., et al. (2013). The efficacy of Chinese herbal medicine as an adjunctive therapy for advanced non-small cell lung cancer: a systematic review and meta-analysis. PLoS One 8 (2), e57604. doi: 10.1371/journal.pone.0057604.

[21] Wang, Q., Jiao, L., Wang, S., Chen, P., Bi, L., Zhou, D., et al. (2018). Maintenance chemotherapy with chinese herb medicine formulas vs. with placebo in patients with advanced non-small cell lung cancer after first-line chemotherapy: a multicenter, randomized, double-blind trial. Front. Pharmacol. 9, 1233. doi: 10.3389/fphar.2018.01233.

[22] Chan A, Tan HL, Ching TH, Tan HC. Clinical outcomes for cancer patients using complementary and alternative medicine. Altern Ther Health Med. 2012; 18 (1): 12-7.

[23] Shah NT, Kris MG, Pao W, Tyson LB, Pizzo BM, Heinemann $\mathrm{MH}$, et al. Practical management of patients with non-small- cell lung cancer treated with gefitinib. J Clin Oncol. 2005; 23 (1): 165-74.

[24] Jackman DM, Miller VA, Cioffredi LA, Yeap BY, Jänne PA, Riely GJ, et al. Impact of epidermal growth factor receptor and KRAS mutations on clinical outcomes in previously untreated non-small cell lung cancer patients: results of an online tumor registry of clinical trials. Clin Cancer Res. 2009; 15 (16): 5267-73.

[25] E. Giovannetti, P. A. Zucali, G. J. Peters et al., "Association of polymorphisms in AKT1 and EGFR with clinical outcome and Journal of Oncology 13toxicity in non-small cell lung cancer patients treated with gefitinib," Molecular Cancer: erapeutics, vol. 9, no. 3, pp. 581-593, 2010.

[26] J. P. A. Ioannidis and T. A. Trikalinos, “'e appropriateness of asymmetry tests for publication bias in meta-analyses: a largesurvey," Canadian Medical Association Journal, vol. 176, no. 8, pp. 1091-1096, 2007. 